\title{
Coherence control for qubits is
}

\author{
Karen M. Fonseca-Romero ${ }^{1}$, Sigmund Kohler *, Peter Hänggi \\ Institut für Physik, Universität Augsburg, Universitätsstraße 1, D-86135 Augsburg, Germany
}

Received 15 July 2003; accepted 17 September 2003

\begin{abstract}
We study the influence of an external driving field on the coherence properties of a qubit subject to bit-flip noise. In the presence of driving, two paradigmatic cases are considered: (i) a field that results for a suitable choice of the parameters in so-called coherent destruction of tunneling and (ii) one that commutes with the static qubit Hamiltonian. In each case, we give for high-frequency driving a lower bound for the coherence time. This reveals the conditions under which the external fields can be used for coherence stabilization.
\end{abstract}

(C) 2003 Elsevier B.V. All rights reserved.

PACS: 03.65.Yz; 03.67.Pp

Keywords: Decoherence; Quantum computation; Driven systems

\section{Introduction}

The experimental realization of one-qubit gates in solid state setups [1,2] and two-qubit gates in ion traps $[3,4]$ and Josephson junctions [5] has demonstrated that these systems provide remarkable coherence properties although the goal of $10^{-5}$ errors per gate operation [6] has not yet been achieved experimentally. Theoretical studies of decoherence of two-level systems [7] have been extended to gate operations in the presence of an environment in [8-12]. The unavoidable coupling to external degrees of freedom and the thereby caused decoherence still presents a main obstacle for the realization of a quantum computer. Thus, proposals for the stabilization of the coherence of qubits are particularly welcome.

A variety of suggestions in this direction relies on the control of coherence by the influence of external fields. In particular, it has been proposed to use the physics of the so-called coherent destruction of tunneling (CDT) for that purpose. CDT has originally been discovered in

\footnotetext{
Dedicated to the 60th birthday of Ulrich Weiss.

* Corresponding author. Fax: +49-821-598-3222.

E-mail address: sigmund.kohler@physik.uni-augsburg.de (S. Kohler).

${ }^{1}$ On leave of absence from Universidad Nacional de Colombia.
}

the context of tunneling in a driven bistable potential $[13,14]$. There, it has been found that a particle which is initially in the, say, left well of a symmetric bistable potential, can be prevented from tunneling by the purely coherent influence of an oscillating driving field. This effect is stable against dissipation in the sense that the ac field also decelerates the dissipative transitions from the left to the right well [15-17] thereby stabilizing coherence. For a preparation in a delocalized superposition, it has been found numerically [10] that the coherent dynamics is not suppressed.

A different proposal for coherence stabilization is the application of a sequence of $\pi$-pulses that flip the sign of the qubit-bath coupling operator resulting in a so-called dynamical decoupling (DD) of the qubit from the bath $[18,19]$. A drawback of this scheme is the fact that it eliminates only noise sources with a frequency below the repetition rate of the pulses. This clearly causes practical limitations. However, these limitations may be circumvented by using a related scheme based on continuouswave driving, i.e. one with a harmonic time-dependence, which allows higher driving frequencies.

In this paper we investigate the coherence properties of a qubit coupled to an ohmic environment which we model by a spin-boson Hamiltonian [20]. We consider two different types of harmonic driving: first, one that 
leads to coherent destruction of tunneling and, second, one that corresponds to a continuous wave version of the dynamical decoupling pulses. In Section 2 we review the spin-boson model for weak dissipation and derive in Section 3 a Markovian formalism for driven dissipative qubits in the form of a basis-free master equation. The global decoherence will be qualified by an upper limit for the decoherence rate that already gives a reliable estimate for the actual value. Subsequently, in Sections 4 and 5 , we study the coherence control by oscillating external fields.

\section{Qubit with bit-flip noise}

The entire system of the qubit and the environmental degrees of freedom is described by the microscopic Hamiltonian

$H=H_{\mathrm{qb}}+H_{\text {coupl }}+H_{\text {bath }}$,

where the qubit is formed by a two-state system with a level splitting $\hbar \Delta$, thus

$H_{\mathrm{qb}}=\frac{\hbar \Delta}{2} \sigma_{z}$.

Below, we will in addition consider an oscillating driving field acting on the qubit. The environment is modeled by a bath of harmonic oscillators which couple linearly to the qubit

$H_{\mathrm{bath}}=\sum_{i} \hbar \omega_{i} a_{i}^{\dagger} a_{i}$

$H_{\text {coupl }}=\frac{1}{2} \sigma_{x} \sum_{i} \hbar c_{i}\left(a_{i}^{\dagger}+a_{i}\right)$,

where $\omega_{i}$ are the oscillator frequencies and $\hbar c_{i}$ the qubitbath coupling energies. The bath couples to the qubit operator $\sigma_{x}$ thereby inducing bit flips, i.e. incoherent transitions between the ground state and the excited state of the qubit.

We complete the model by choosing an initial condition $\rho_{\text {tot }}\left(t_{0}\right)$, of the Feynman-Vernon type, i.e., we assume that at time $t=t_{0}$ the bath is in thermal equilibrium and uncorrelated with the qubit

$\rho_{\text {tot }}\left(t_{0}\right)=\rho\left(t_{0}\right) \otimes R_{\text {bath, eq }}$,

where $\rho$ is the reduced density operator of the qubit and $R_{\text {bath, eq }} \propto \exp \left(-\beta H_{\text {bath }}\right)$ is the canonical ensemble of the bath at the inverse temperature $\beta=1 / k_{\mathrm{B}} T$.

\subsection{Dissipative qubit dynamics}

From the Liouville-von Neumann equation $\mathrm{i} \hbar \dot{\rho}_{\text {tot }}=$ $\left[H, \rho_{\text {tot }}\right]$ for the total density operator one obtains for the reduced density operator of the qubit by standard techniques, the Markovian weak-coupling master equation

$$
\begin{aligned}
\dot{\rho}=- & \frac{\mathrm{i}}{\hbar}\left[H_{\mathrm{qb}}, \rho\right]-\frac{1}{4} \int_{0}^{\infty} \mathrm{d} \tau\left(\mathscr{S}(\tau)\left[\sigma_{x},\left[\tilde{\sigma}_{x}(t-\tau, t), \rho\right]\right]\right. \\
& \left.+\mathscr{A}(\tau)\left[\sigma_{x},\left\{\tilde{\sigma}_{x}(t-\tau, t), \rho\right\}\right]\right),
\end{aligned}
$$

where $\{A, B\}=A B+B A$ stands for the anticommutator. The notation $\tilde{X}\left(t, t^{\prime}\right)$ is a shorthand for $U^{\dagger}\left(t, t^{\prime}\right) X U\left(t, t^{\prime}\right)$ with $U$ being the propagator for the coherent qubit dynamics. Note that for later use, we have written the master equation already in a form which is also valid in the case of an explicit time-dependence of the qubit Hamiltonian. The dissipative equation of motion (6) depends on the bath through the symmetric and antisymmetric correlation functions of the bath operator $\mathscr{B}(t)=\sum_{i} c_{i}\left(a_{i}^{\dagger} \exp \left(\mathrm{i} \omega_{i} t\right)+a_{i} \exp \left(-\mathrm{i} \omega_{i} t\right)\right)$, given by

$$
\begin{aligned}
\mathscr{S}(\tau) & =\frac{1}{2}\langle\{\mathscr{B}(\tau), \mathscr{B}(0)\}\rangle \\
& =\frac{1}{\pi} \int_{0}^{\infty} \mathrm{d} \omega J(\omega) \operatorname{coth}(\hbar \omega \beta / 2) \cos (\omega \tau),
\end{aligned}
$$

$$
\begin{aligned}
\mathscr{A}(\tau) & =\frac{1}{2}\langle[\mathscr{B}(\tau), \mathscr{B}(0)]\rangle \\
& =-\frac{\mathrm{i}}{\pi} \int_{0}^{\infty} \mathrm{d} \omega J(\omega) \sin (\omega \tau),
\end{aligned}
$$

respectively. The angular brackets $\langle\cdots\rangle$ denote the average with respect to the thermal equilibrium of the bath. We have introduced for the qubit-bath coupling the spectral density

$J(\omega)=\pi \sum_{i} c_{i}^{2} \delta\left(\omega-\omega_{i}\right)$.

If the bath modes are dense, $J(\omega)$ becomes a smooth function. Within the present work, we will consider the case of Ohmic dissipation where $J(\omega)=2 \pi \alpha \omega \exp (-\omega /$ $\left.\omega_{\mathrm{c}}\right)$ and $\alpha$ is a dimensionless measure for the dissipation strength. The so-called cutoff frequency $\omega_{\mathrm{c}}$ is assumed to be the highest frequency of the bath. If the cutoff frequency is much larger than all relevant energy scales, the antisymmetric correlation function $\mathscr{A}(\tau)$ can be replaced by $2 \pi \mathrm{i} \alpha \delta^{\prime}(\tau)$. Then, for the integral in the master equation (6), the part containing $\mathscr{A}(\tau)$ can be evaluated after integrating by parts to read

$\frac{\pi \alpha}{4}\left[\sigma_{x},\left\{\left[H_{\mathrm{qb}} / \hbar, \sigma_{x}\right], \rho\right\}\right]$.

To bring the master equation (6) into a more explicit form, we insert the Heisenberg operator

$\tilde{\sigma}_{x}(t-\tau, t)=\sigma_{x} \cos (\Delta \tau)+\sigma_{y} \sin (\Delta \tau)$,

which is readily derived from its definition together with the qubit Hamiltonian (2). Performing the integration over $\tau$ and $\omega$ and neglecting renormalization effects, which are small provided that $\alpha \ln \left(\omega_{\mathrm{c}} / \Delta\right) \ll 1$, yields for $\Delta \ll \omega_{\mathrm{c}}$, the Markovian master equation 
$\dot{\rho}=-\frac{\mathrm{i}}{\hbar}\left[H_{\mathrm{qb}}, \rho\right]-\frac{\Gamma}{4}\left[\sigma_{x},\left[\sigma_{x}, \rho\right]\right]+\mathrm{i} \frac{\pi \alpha \Delta}{4}\left[\sigma_{x},\left\{\sigma_{y}, \rho\right\}\right]$.

We will find below that the relaxation and dephasing processes are determined by the rate [20]

$\Gamma=\frac{1}{2} \mathscr{S}(\Delta)$

where

$\mathscr{S}(\omega)=2 \pi \alpha \omega \operatorname{coth}\left(\frac{\hbar \omega \beta}{2}\right)$

is the power spectrum of the bath fluctuations, i.e. the Fourier transformed of the symmetric bath correlation function (7). This master equation is a basis-free version of a Bloch-Redfield or Floquet-Markov master equation [21,22]. Such an operator notation is preferable to a decomposition into the qubit's eigenbasis for ease of notation and, moreover, since it allows a more elegant computation of expectation values. The interpretation of (12) is that the first term of the right-hand side is responsible for the coherent dynamics, while the second and third term correspond to decoherence and relaxation.

For the description of the dynamics of a single qubit, it is convenient to map the density operator to the Bloch vector $\vec{s}=\operatorname{tr}(\vec{\sigma} \rho)$. It is straightforward to derive for the Bloch vector from the master equation (12) the inhomogeneous linear equation of motion

$\dot{\vec{s}}=-M \vec{s}+\vec{b}$,

where

$M=\left(\begin{array}{ccc}0 & -\Delta & 0 \\ \Delta & \Gamma & 0 \\ 0 & 0 & \Gamma\end{array}\right), \quad \vec{b}=\left(\begin{array}{c}0 \\ 0 \\ -\pi \alpha \Delta\end{array}\right)$.

In the weak dissipation limit, $\Gamma \ll \Delta$, the matrix $M$ has the eigenvalues

$\Gamma, \quad \frac{1}{2} \Gamma \pm \mathrm{i} \Delta$.

While the first eigenvalue describes the decay of the population of the excited state, the other two correspond to damped oscillations of the off-diagonal density matrix elements in the basis eigenbasis of the qubit Hamiltonian. This justifies for $\Gamma$ the designation relaxation rate and the definition of the decoherence rate $\Gamma_{\phi}=\Gamma / 2$. For larger systems there is room for several relaxation (purely real eigenvalues) and decoherence rates (complex eigenvalues with non-vanishing imaginary parts).

\subsection{Entropy production}

Relaxation and decoherence are non-unitary processes, i.e. processes which transform a pure state into a mixed state. Since a quantum computer relies fundamentally on the coherence of the time evolution, such processes put an essential limitation and their influence has to be minimized. Thus, in order to follow the process of coherence loss, it is desirable to define proper coherence measures which preferably are independent of the chosen basis. A natural possibility that comes to mind is the Shannon entropy $\operatorname{tr}(\rho \ln \rho)$. However, this measure is sometimes inconvenient due to the appearance of the logarithm. Therefore, it is common to use instead the "linear entropy" [23-25]

$S=1-\operatorname{tr}\left(\rho^{2}\right)=\frac{1}{2}(1-\vec{s} \cdot \vec{s})$,

which is a good approximation for the Shannon entropy for almost pure states and is closely related to the socalled purity $\operatorname{tr}\left(\rho^{2}\right)$. The history of this kind of measure is long, and can be traced back to at least 1939 [23]. The linear entropy possesses a convenient physical interpretation: Suppose that $\rho$ describes an incoherent mixture of $n$ orthogonal states with equal probability, then $\operatorname{tr}\left(\rho^{2}\right)$ reads $1 / n$. For a single qubit the maximum linear entropy is therefore $1 / 2$. It is zero if and only if $\rho$ describes a pure state.

The decoherence rate is well described by the linearized entropy production

$\dot{S}=-2 \operatorname{tr}(\rho \dot{\rho})=-\vec{s} \cdot \dot{\vec{s}}$,

which follows directly from the master equation, respectively, from the equation of motion for the Bloch vector. Obviously, the coherent dynamics given by the first commutator in the master equation (12) does not increase the entropy.

In the context of quantum computing, we are mainly interested in the entropy production for (almost) pure states, i.e. the initial entropy production which is determined by the eigenvalues (17) of the matrix $M$. Since these eigenvalues are invariant under unitary transformations, they represent a global measure for the influence of the dissipation. In particular, they are independent of the choice of the basis. In the worst case, the decoherence is determined by the eigenvalue with the largest real part.

For the driven system considered below, the matrix becomes time-dependent and, consequently, the eigenvalues (17) must be replaced by the eigenvalues of a Floquet equation. Their computation, however, might be quite cumbersome. Therefore, a more convenient measure which is also applicable to driven systems is the sum $\gamma$ of all eigenvalues which can be computed without explicit diagonalization by the trace of the matrix $M$,

$\gamma \equiv \operatorname{tr} M=2 \Gamma$

Since $\gamma$ is real and larger than all real parts of the eigenvalues (which are positive), it represents an upper bound for the decoherence rate. Moreover, for the 
present case, the largest real part of an eigenvalue is at least $\gamma / 3$ and, thus, we can conclude that $\gamma$ gives the correct order of magnitude for the decoherence rate of the most fragile initial state.

A further related, but more probabilistic measure, that characterizes the loss of coherence, is the average $\dot{S}$ over all pure initial states of the entropy production and reads

$\Gamma_{\mathrm{av}} \equiv\langle\dot{S}(0)\rangle_{\mathrm{av}}=\frac{\gamma}{3}$.

\section{Dissipation and driving}

To study the controllability of the decoherence, we generalize the master equation to the case where a timedependent Hamiltonian

$H_{\mathrm{D}}(t)=H_{1} f(t)$

acts on the qubit. We assume that time-dependence $f(t)$ is periodic with the driving period $T=2 \pi / \Omega$ and has zero average. Then, according to the Floquet theorem [26], the corresponding propagator can always be written in the form

$U\left(t, t^{\prime}\right)=U_{\mathrm{P}}\left(t, t^{\prime}\right) U_{\mathrm{F}}\left(t-t^{\prime}\right)$,

where $U_{\mathrm{P}}\left(t, t^{\prime}\right)=U_{\mathrm{P}}\left(t+T, t^{\prime}\right)$ obeys the time-periodicity of the driving field. The Floquet propagator $U_{\mathrm{F}}$ depends only on the time difference and contains non-adiabatic phases which emerge during the propagation over one driving period [27]. Since $U_{\mathrm{P}}$ is time-periodic, the longtime dynamics of the driven qubit is entirely determined by the one-period propagator $U_{\mathrm{F}}(T)$.

Inserting the propagator (23) into the master equation (12) results after the $\tau$-integration in a dissipative kernel that still depends periodically on the final time $t$. However, since we consider the case of fast driving, it is possible to separate time-scales and replace the rapidly oscillating operators in the master equation by their time-averages. In doing so, we arrive at $[17,22]$

$$
\begin{aligned}
\dot{\rho}= & -\frac{\mathrm{i}}{\hbar}\left[H_{\mathrm{qb}}+H_{\mathrm{D}}(t), \rho\right]-\frac{1}{4}\left[\sigma_{x},[Q, \rho]\right] \\
& +\mathrm{i} \frac{\pi \alpha \Delta}{4}\left[\sigma_{x},\left\{\sigma_{y}, \rho\right\}\right],
\end{aligned}
$$

where the difference with respect to the master equation (12) for the static case comes from replacing in the second commutator $\Gamma \sigma_{x}$ by the operator

$$
\begin{aligned}
Q= & \frac{1}{T} \int_{0}^{T} \mathrm{~d} t \int_{0}^{\infty} \mathrm{d} \tau \mathscr{S}(\tau) U_{\mathrm{F}}^{\dagger}(\tau) U_{\mathrm{P}}^{\dagger}(t-\tau, t) \\
& \times \sigma_{x} U_{\mathrm{P}}(t-\tau, t) U_{\mathrm{F}}(\tau) .
\end{aligned}
$$

The master equation (24) for the driven, dissipative qubit reflects the close resemblance to the master equation (12) valid in the static case. The last term in (24) is not modified by the driving. The reason for this is that the driving enters linearly in (10) and, consequently, vanishes in the average over the driving period. However, besides the explicit presence of the time-dependent Hamiltonian in the coherent contribution, also the dissipative part has acquired a change: the coupling operator $\sigma_{x}$ is now replaced by an operator $Q$ that depends on the qubit propagator in the presence of the driving. As a consequence, we expect in the case of strong driving fields not only a modification of the coherent dynamics, but also of the coherence properties. In the following sections, we will investigate two typical types of driving: as a result, we find that they can alter the coherence times rather significantly.

\section{Coherent destruction of tunneling}

As a first example for the significant influence of a driving field, we investigate the qubit under the influence of the Hamiltonian

$H_{\mathrm{D}}(t)=A \sigma_{x} \cos (\Omega t)$,

which couples like the bath to the qubit by the operator $\sigma_{x}$ and, thus, commutes with the qubit-bath coupling. Such a time-dependent field causes already interesting effects for the coherent qubit dynamics that we will briefly review before discussing decoherence.

To sketch the coherent dynamics of the driven qubit, we first transform the Hamiltonian $H_{\mathrm{qb}}+H_{\mathrm{D}}(t)$ by the unitary operator

$U_{0}(t)=\exp \left(-\mathrm{i} \frac{A \sigma_{x}}{\hbar \Omega} \sin (\Omega t)\right)$.

This transformation defines the interaction picture with respect to $H_{\mathrm{D}}(t)$ and results in the likewise $T$-periodic Hamiltonian

$$
\begin{aligned}
\tilde{H}_{\mathrm{qb}}(t)= & \frac{\hbar \Delta}{2}\left\{\sigma_{z} \cos \left(\frac{2 A}{\hbar \Omega} \sin (\Omega t)\right)\right. \\
& \left.+\sigma_{y} \sin \left(\frac{2 A}{\hbar \Omega} \sin (\Omega t)\right)\right\} .
\end{aligned}
$$

The corresponding Schrödinger equation cannot be integrated exactly since $\tilde{H}_{\mathrm{qb}}(t)$ does not commute with itself at different times and, thus, time-ordering has to be taken into account. We restrict ourselves to an approximate solution and neglect corrections of the order $\Delta^{2}$. Within this approximation, the propagator is simply given by the exponential of the integral of the timedependent Hamiltonian. This consists of two parts: First, there is the time-average of $\tilde{H}_{\mathrm{qb}}(t)$ which determines the leading contribution to the Floquet propagator $U_{\mathrm{F}}(t)$. The second part comprises terms that oscillate with the driving period. For a high-frequency driving with $\Omega \gg \Delta$, this latter contribution can be neglected since the periodic part of the propagator is dominated by the contribution $U_{0}$ coming directly from the driving. This 
approach is equivalent to a perturbational computation of Floquet states [28,29] as has been shown in [30].

Finally, the qubit propagator in the interaction picture defined by (27), is determined by the time average of the Hamiltonian (28) which reads

$\bar{H}_{\mathrm{qb}}=\frac{\hbar \Delta_{\mathrm{eff}}}{2} \sigma_{z}$.

This static approximation to the driven qubit Hamiltonian is of the same form as the original Hamiltonian (2), but with the tunneling matrix element renormalized according to

$\Delta \rightarrow \Delta_{\text {eff }}=J_{0}(2 A / \hbar \Omega) \Delta$.

Here, $J_{0}$ is the zeroth-order Bessel function of the first kind. Thus, within this high-frequency approximation, the entire propagator (23) for the qubit in the Schrödinger picture reads

$U(t, 0)=\exp \left(-\mathrm{i} \frac{A}{\hbar \Omega} \sin (\Omega t) \sigma_{x}\right) \exp \left(-\mathrm{i} \frac{\Delta_{\text {eff }} t}{2} \sigma_{z}\right)$.

Of particular interest are now driving parameters for which $2 A / \hbar \Omega$ corresponds to a zero of the Bessel function $J_{0}$, i.e. for which $\Delta_{\text {eff }}$ vanishes. Then the one-period propagator $U_{\mathrm{F}}(T)$, becomes the identity. Or in other words: the long-time dynamics is suppressed. The dynamics within the driving period requires a closer look at the periodic propagator $U_{\mathrm{P}}$ : for an initial preparation in an eigenstate of $\sigma_{x}$, it contributes only a global phase, such that the dynamics as a whole is suppressed also within the driving period. This effect of suppressing the time-evolution by the purely coherent influence of an external field has been investigated first in the context of driven tunneling $[13,14]$ and is named "coherent destruction of tunneling" (CDT). ${ }^{2}$ However, for a preparation other than an eigenstate of $\sigma_{x}$, the periodic propagator $U_{\mathrm{P}}$ will still cause a non-trivial dynamics within the driving period. This fact has been found numerically in [10].

There is also a significant influence of the driving (26) on the dissipative dynamics of a particle with an initial preparation in an eigenstate of $\sigma_{x}$ : besides slowing down the coherent time evolution, also the dissipative transitions are decelerated, i.e. the rate for the dissipative transitions between the wells of a bistable system becomes lower. In the following, we investigate decoherence and dissipation under such a CDT driving without the restriction to a specific preparation.

In order to evaluate the coefficients of the master equation (24) valid in the driven case, we have to compute the operator $Q$. This requires an explicit expression

\footnotetext{
${ }^{2}$ Although we consider here also parameters for which the dynamics is not entirely suppressed, we will refer to a driving of the form (26) as "CDT driving".
}

for the propagator $U\left(t, t^{\prime}\right)$ starting at time $t^{\prime}$ and, thus, we have to consider initial phases. Repeating the calculation from the beginning of this section yields

$$
\begin{aligned}
U(t-\tau, t)= & \exp \left(-\mathrm{i} \frac{A}{\hbar \Omega}[\sin \Omega(t-\tau)-\sin \Omega t] \sigma_{x}\right) \\
& \times \exp \left(-\mathrm{i} \frac{\Delta_{\text {eff }} \tau}{2} \sigma_{z}\right),
\end{aligned}
$$

where the first factor is the time-periodic part $U_{\mathrm{P}}(t-\tau, t)$ while the second factor determines the long-time dynamics. Since $U_{\mathrm{P}}$ commutes with $\sigma_{x}$, only the Floquet propagator $U_{\mathrm{F}}$ is relevant for the operator (25). After performing the time and the frequency integration we find

$Q_{\mathrm{CDT}}=\frac{1}{2} \mathscr{S}\left(\Delta_{\mathrm{eff}}\right) \sigma_{x}$.

Thus, the master equation is of the same form as in the undriven case, Eq. (12), but with $\Gamma$ replaced by

$$
\Gamma_{\mathrm{CDT}}=\frac{1}{2} \mathscr{S}\left(\Delta_{\mathrm{eff}}\right)=\Gamma J_{0}(2 A / \hbar \Omega) \frac{\operatorname{coth}\left(\beta \hbar \Delta_{\mathrm{eff}} / 2\right)}{\operatorname{coth}(\beta \hbar \Delta / 2)} .
$$

Since the spectral density $S(\omega)$ increases monotonically with the frequency $\omega$ and, moreover, the Bessel function $J_{0}(x)<1$ for $x>0$, dissipation and decoherence become smaller due to the driving.

Again, we employ the trace of the matrix $M$ appearing in the equation of motion for the Bloch vector as a criterion for the global decoherence strength and find

$\gamma_{\mathrm{CDT}}=\mathscr{S}\left(\Delta_{\mathrm{eff}}\right)$,

which represents within the high-frequency approximation an upper limit for the decoherence rates. For high temperatures, $\beta \hbar \Delta_{\text {eff }} \ll 1$, the coth in the power spectrum (14) can be replaced by the inverse of its argument. This defines the classical limit where the decoherence rate (35) becomes $4 \pi \alpha k_{\mathrm{B}} T / \hbar$, i.e. independent of the effective tunnel splitting $\Delta_{\text {eff }}$. In the low temperature limit $\beta \hbar \Delta_{\text {eff }} \gg 1$, the coth becomes unity and, thus,

$\gamma_{\mathrm{CDT}} \approx \gamma J_{0}(2 A / \hbar \Omega)$,

which means that decoherence becomes smaller by a factor $J_{0}(2 A / \hbar \Omega)$. This reduction of decoherence is brought about by the fact that the driving (26) decelerates the long time dynamics of the qubit. Thereby, the frequencies which are relevant for the decoherence are shifted to a range where the spectral density of the bath is lower. Consequently, the influence of the bath is diminished. Since $\gamma_{\mathrm{CDT}}$ represents an upper bound for the decoherence rate, this demonstrates that coherence stabilization via CDT is a phenomenon that is independent of the initial state.

It should be noted, however, that the coherent dynamics is slowed down by the same factor as the decoherence, cf. Eqs. (30) and (36). Thus, if for a specific 
application, the figure of merit is the number of coherent oscillations, the present coherence stabilization scheme may not prove useful. For resonant driving, this unfavorable situation can change for certain preparations, see Fig. 2 in [10].

\section{Dynamical decoupling}

A recently proposed mechanism for coherence stabilization is the so-called dynamical decoupling [18]. This scheme employs sequences of $\pi$-pulses that flip the sign of the operator that couples the qubit to the bath operators. The basic idea originates from the suppression of spin diffusion in nuclear magnetic resonance experiments [31,32] and has become an established technique in that area [33]. In the present case where the bath couples to the operator $\sigma_{x}$ [cf. Eq. (4)], such a transformation is, e.g. induced by the Hamiltonian $\hbar \omega_{\mathrm{R}} \sigma_{z}$ for a pulse duration $\pi / \omega_{\mathrm{R}}$. Since the corresponding propagator is a function of the qubit Hamiltonian, the coherent dynamics is not altered. Besides the prospective benefits of such a control scheme, there is also a number of possible drawbacks that the application of $\pi$-pulses might cause: For a driven system, there is always the possibility of unwanted off-resonant transitions [34], especially in the case of sharp pulses. A more practical limitation is the fact that only noise with frequencies below the pulse repetition rate can be eliminated in such a way.

These disadvantages can be overcome partially by applying a continuous wave version of the dynamical decoupling scheme, i.e. a driving of the form

$H_{\mathrm{D}}=A \sigma_{z} \cos (\Omega t)$

for which the available frequency range is larger. This constitutes our second example of a driving field influencing considerably the qubit decoherence. In contrast to the driving Hamiltonian employed in the previous section, the present one commutes with the static qubit Hamiltonian and, consequently, the propagator for the driven qubit can be computed exactly to read

$$
\begin{aligned}
U\left(t, t^{\prime}\right)= & \exp \left(-\mathrm{i} \frac{A}{\hbar \Omega}\left[\sin (\Omega t)-\sin \left(\Omega t^{\prime}\right)\right] \sigma_{z}\right) \\
& \times \exp \left(-\mathrm{i} \Delta \sigma_{z}\left(t-t^{\prime}\right)\right) .
\end{aligned}
$$

Again, we have written the propagator in the form (23) which is suitable for simplifying the master equation (24) Inserting this into the expression (25) results for $\Delta \ll \Omega$ in the effective coupling operator

$$
\begin{aligned}
Q_{\mathrm{DD}}= & \frac{1}{2} \sigma_{x}\left(J_{0}^{2}(2 A / \hbar \Omega) \mathscr{P}(\Delta)\right. \\
& \left.+2 \sum_{n=1}^{\infty} J_{n}^{2}(2 A / \hbar \Omega) \mathscr{P}(n \Omega)\right) .
\end{aligned}
$$

In order to derive this expression, we have decomposed the exponentials of the trigonometric functions into a Fourier series using the identity $\exp [\mathrm{i} x \sin (\Omega t)]=$ $\sum_{k} J_{k}(x) \exp (\mathrm{i} k \Omega t)$, where $J_{k}$ is the $k$ th-order Bessel function of the first kind [35]. Like in the previous cases, the effective coupling operator $Q_{\mathrm{DD}}$ is proportional to $\sigma_{x}$ and, thus, the master equation is again of the form (12). The only change for the dissipative dynamics is the replacement of $\Gamma$ by

$$
\begin{aligned}
\Gamma_{\mathrm{DD}}= & \Gamma\left\{J_{0}^{2}(2 A / \hbar \Omega)\right. \\
& \left.+2 \sum_{n=1}^{\infty} \frac{n \Omega}{\Delta} \frac{\tanh (\hbar \Delta \beta / 2)}{\tanh (n \hbar \Omega \beta / 2)} \mathrm{e}^{-n \Omega / \omega_{\mathrm{c}}} J_{n}^{2}(2 A / \hbar \Omega)\right\} .
\end{aligned}
$$

The decoherence rate in this case depends on the spectral density of the bath at multiples of the driving frequency which may be larger than the cutoff frequency $\omega_{\mathrm{c}}$. The $\pi$ pulses applied in the original version [18] of dynamical decoupling, correspond for a continuous driving to a field amplitude such that $2 A / \hbar \Omega$ equals the first zero of the Bessel function $J_{0}$, i.e. assumes a value $2.404825 \ldots$ Then only the sum in Eq. (40) contributes to the decoherence rate $\Gamma_{\mathrm{DD}}$. If now the driving frequency is larger than the cutoff of the spectral density, $\Omega>\omega_{\mathrm{c}}$, the decoherence rate is considerably reduced: For low temperatures, $1 / \beta \ll \hbar \Delta$, the hyperbolic tangent in the decoherence rate (40) become unity and each contribution is weighted by a possibly large factor $n \Omega / \Delta$. In the high-temperature limit $1 / \beta \gg \hbar \Omega$, we use $\tanh x \approx x$ and find that the dependence of the prefactor on $n \Omega$ cancels. This means that the dynamical decoupling scheme is especially useful for high temperatures. The physical reason for this is that the driving shifts the qubit dynamics towards high frequencies where the thermal occupation of the bath modes is negligible.

To assess the coherence stabilization originating from dynamical decoupling, we define for the coherence stabilization the rather conservative measure

$\eta=\frac{\Gamma / 2}{\gamma_{\mathrm{DD}}}$,

i.e. the ratio between the lowest decoherence rate without driving and the largest decoherence rate with driving, cf. Eqs. (17) and (20). For parameters such that $\eta>1$, it is granted that the driving stabilizes the coherence independent of the initial state. If $\eta>1 / 4$, we still can conclude that the coherence is improved in the average over all possible initial states.

Fig. 1 compares the coherence stabilization $\eta$ as a function of the driving frequency for $2 A / \hbar \Omega=2.4$, i.e. close to a zero of the Bessel function $J_{0}$. It reveals that for driving frequencies well below the cutoff, the driving rather spoils the coherence. This improves with 


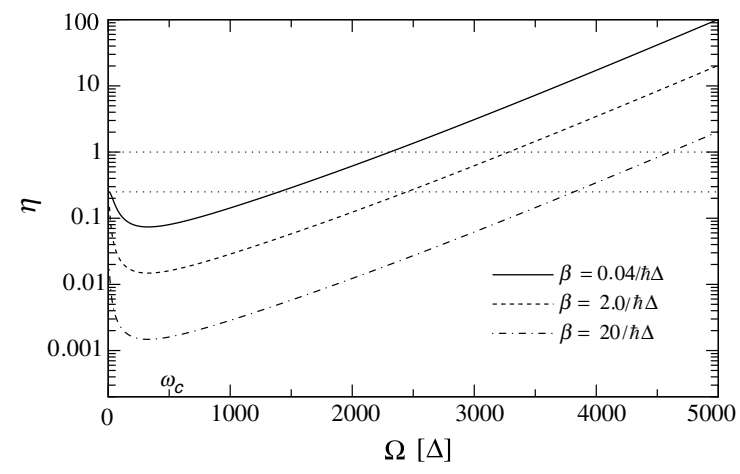

Fig. 1. Coherence stabilization factor $\eta$ as a function of the driving frequency for dynamical decoupling for different temperatures. The cutoff frequency is $\omega_{\mathrm{c}}=500 \Delta, 2 A / \hbar \Omega=2.4$, and the dissipation strength is $\alpha=0.01$. The dotted horizontal lines mark the values above which the coherence is improved on average $(\eta>1 / 4)$ and for an arbitrary initial condition $(\eta>1)$, respectively.

increasing driving frequency and, finally, for a highfrequency driving, $\eta$ becomes much larger than unity corresponding to a significant coherence stabilization. The data demonstrate the usefulness of dynamical decoupling for high temperatures.

\section{Conclusions}

We have investigated the coherence properties of a qubit weakly coupled to a harmonic oscillator bath under the influence of an external driving field. Two types of driving have been taken into account: one that can cause coherent destruction of tunneling and another that corresponds to $\pi$-pulses that invert the sign of the qubit-bath coupling operator. To estimate the decoherence, we have derived an upper bound for the decoherence rate. As a result, we have found that both types of driving can enhance the coherence properties of the qubit significantly, independent of the preparation.

In the case of CDT we have found that the main effect comes from the fact that the driving shifts the coherent long-time dynamics of the qubit towards lower frequencies where the spectral density of an ohmic bath is lower and, thus, the effective coupling is weaker. Consequently, decoherence is generally reduced. This is most pronounced at low temperatures. For high temperatures, however, the lower spectral density is counterbalanced by an increasing thermal noise, such that decoherence becomes independent of the driving.

For the continuous-wave dynamical decoupling scheme, we have found that a low-frequency driving is counterproductive. However, once the frequency exceeds the bath cutoff, the coherence properties recover and are finally significantly improved, especially at high temperatures. Since such a dynamical decoupling by a harmonic driving allows higher driving frequencies than the pulsed version, this form of coherence stabilization bears interesting perspectives for applications.

\section{Acknowledgements}

This work has been supported by the research network "Quanteninformation entlang der A8" and the Sonderforschungsbereich 631 of the Deutsche Forschungsgemeinschaft.

\section{References}

[1] D. Vion, A. Aassime, A. Cottet, P. Joyez, H. Pothier, C. Urbina, D. Esteve, M.H. Devoret, Science 296 (2002) 886.

[2] I. Chiourescu, Y. Nakamura, C.J.P. Harmans, J.E. Mooij, Science 299 (2003) 1869.

[3] D. Leibfried, B. DeMarco, V. Meyer, V.D. Lucas, M. Barrett, J. Britton, W.M. Itano, B. Jelenković, C. Langer, T. Rosenband, D.J. Wineland, Nature 422 (2003) 412.

[4] F. Schmidt-Kaler, H. Häffner, M. Riebe, S. Gulde, G.P.T. Lancaster, T. Deuschle, C. Becher, C.F. Roos, J. Eschner, R. Blatt, Nature 422 (2003) 408.

[5] Y.A. Pashkin, T. Yamamoto, O. Astafiev, Y. Nakamura, D.V. Averin, J.S. Tsai, Nature 421 (2003) 823.

[6] A.M. Steane, Fortschr. Phys. 46 (1998) 443.

[7] A.J. Leggett, S. Chakravarty, A.T. Dorsey, M.P.A. Fisher, A. Garg, W. Zwerger, Rev. Mod. Phys. 59 (1987) 1.

[8] D. Loss, D.P. DiVincenzo, Phys. Rev. A 57 (1998) 120.

[9] M. Grifoni, E. Paladino, U. Weiss, Eur. Phys. J. 10 (1999) 719.

[10] M. Thorwart, L. Hartmann, I. Goychuk, P. Hänggi, J. Mod. Opt. 47 (2000) 2905;

M. Thorwart, P. Hänggi, Phys. Rev. A 65 (2002) 012309.

[11] M. Governale, M. Grifoni, G. Schön, Chem. Phys. 268 (2001) 273.

[12] M.J. Storcz, F.K. Wilhelm, Phys. Rev. A 67 (2003) 042319.

[13] F. Grossmann, T. Dittrich, P. Jung, P. Hänggi, Phys. Rev. Lett. 67 (1991) 516.

[14] F. Grossmann, P. Jung, T. Dittrich, P. Hänggi, Z. Phys. B 84 (1991) 315.

[15] T. Dittrich, B. Oelschlägel, P. Hänggi, Europhys. Lett. 22 (1993) 5.

[16] M. Grifoni, M. Sassetti, P. Hänggi, U. Weiss, Phys. Rev. E 52 (1995) 3596.

[17] L. Hartmann, I. Goychuk, M. Grifoni, P. Hänggi, Phys. Rev. E 61 (2000) R4687.

[18] L. Viola, S. Lloyd, Phys. Rev. A 58 (1998) 2733.

[19] L. Viola, E. Knill, S. Lloyd, Phys. Rev. Lett. 82 (1999) 2417.

[20] U. Weiss, Quantum Dissipative Systems, Series in Modern Condensed Matter Physics, vol. 2, World Scientific, Singapore, 1993.

[21] R. Blümel, A. Buchleitner, R. Graham, L. Sirko, U. Smilansky, H. Walter, Phys. Rev. A 44 (1991) 4521.

[22] S. Kohler, T. Dittrich, P. Hänggi, Phys. Rev. E 55 (1997) 300

[23] S. Watanabe, Z. Phys. 113 (1939) 482.

[24] M.C. Nemes, A.F.R. de Toledo Piza, Physica A 137 (1986) 367.

[25] W.H. Zurek, S. Habib, J.P. Paz, Phys. Rev. Lett. 70 (1993) 1187.

[26] N.L. Manakov, V.D. Ovsiannikov, L.P. Rapoport, Phys. Rep. 141 (1986) 319

[27] Y. Aharonov, J. Anandan, Phys. Rev. Lett. 58 (1987) 1593.

[28] J.H. Shirley, Phys. Rev. 138 (1965) B979. 
[29] M. Grifoni, P. Hänggi, Phys. Rep. 304 (1998) 229.

[30] S. Kohler, S. Camalet, M. Strass, J. Lehmann, G.-L. Ingold, P. Hänggi, Chem. Phys. (2004) this issue.

[31] H.Y. Carr, E.M. Purcell, Phys. Rev. 94 (1954) 630.

[32] U. Haeberlen, J.S. Waugh, Phys. Rev. 175 (1968) 453.
[33] C.P. Slichter, Principles of Magnetic Resonance, third ed., Springer Series in Solid State Sciences, vol. 1, Springer, Berlin, 1990.

[34] L. Tian, S. Lloyd, Phys. Rev. A 62 (2000) 050301.

[35] I.M. Gradshteyn, I.S. Ryzhik, Table of Integrals, Series, and Products, fifth ed., Academic Press, San Diego, 1994. 